\title{
Biomechanical In Vitro - Stability Testing on Human Specimens of a Locking Plate System Against Conventional Screw Fixation of a Proximal First Metatarsal Lateral Displacement Osteotomy
}

\author{
Heino Arnold ${ }^{*}$, , Christina Stukenborg-Colsman ${ }^{2}$, Christof Hurschler ${ }^{3}$, Frank Seehaus ${ }^{3}$, \\ Evgenij Bobrowitsch ${ }^{3}$ and Hazibullah Waizy ${ }^{2}$
}

\author{
${ }^{1}$ Orthopädisch - Unfallchirurgische Praxisklinik, Orthopädisches Zentrum Fichtelgebirge, Bahnhofstraße 10, 95111 \\ Rehau, Germany \\ ${ }^{2}$ Orthopädische Klinik der MHH im Diakoniekrankenhaus Annastift, Anna-von-Borries-Straße 1-7, 30625 Hannover- \\ Kleefeld. Germany \\ ${ }^{3}$ Orthopädische Klink der Medizinischen Hochschule Hannover, Labor für Biomechanik und Biomaterialien, Anna-von- \\ Borries-Str. 1-7, 30625 Hannover, Germany
}

\begin{abstract}
Introduction: The aim of this study was to examine resistance to angulation and displacement of the internal fixation of a proximal first metatarsal lateral displacement osteotomy, using a locking plate system compared with a conventional crossed screw fixation.

Materials and Methodology: Seven anatomical human specimens were tested. Each specimen was tested with a locking screw plate as well as a crossed cancellous srew fixation. The statistical analysis was performed by the Friedman test. The level of significance was $\mathrm{p}=0.05$.

Results: We found larger stability about all three axes of movement analyzed for the PLATE than the crossed screws osteosynthesis (CSO). The Friedman test showed statistical significance at a level of $p=0.05$ for all groups and both translational and rotational movements.

Conclusion: The results of our study confirm that the fixation of the lateral proximal first metatarsal displacement osteotomy with a locking plate fixation is a technically simple procedure of superior stability.
\end{abstract}

Keywords: Biomechanical stability testing, metatarsal osteotomy, locking plate system.

\section{INTRODUCTION}

Although the incidence data on the occurence of hallux valgus deformity found in literature vary, it can still considered as collectively a common occurance, with between $2 \%$ (children) and 49,5\% (European adults) of the population being affected [1-3]. An internal fixation is required at least in adults to correct the hallux deformity permanently, an operation that has been described in more than 130 variations over the last 100 years [4]. The choice to operate and what technique to apply depends on the severity of the deformity $[5,6]$. In accordance with current guidelines proximal operations of the first metatarsal are reserved for severe deformities with pronounced intermetatarsal - I angles over $15^{\circ}-20^{\circ}$ and hallux valgus - angles over $40^{\circ}$ [7].

A problem of a proximal intervention is the achievement of a biomechanically competent internal fixation in order to realize an early functional treatment, as well as to avoid the possible drawbacks associated with a plaster cast

*Address correspondence to this author at the Orthopädisch - Unfallchirurgische Praxisklinik, Orthopädisches Zentrum Fichtelgebirge, Bahnhofstraße 10, 95111 Rehau, Germany; Tel: ++49928381097; Fax: ++49928381099;

E-mail: orthopaedie_rehau@t-online.de immobilisation of longer duration. The commonly used screw fixation is biomechanically incapable of bearing load and thus not considered weightbearing [8]. In theory, this results in an risk of a secondary correction loss, which may have particularly serious consequences when considering the long lever acting at the distal first metatarsal after a proximal osteotomy. This problem of possible secondary loss of correction has led to the development of advanced locking plate systems with the aim of allowing the partial load bearing without loss of correction, even after a proximal first metatarsal proceedure.

The aim of this study was thus to examine resistance to angulation and displacement of the internal fixation of a proximal first metatarsal lateral displacement osteotomy as described by Thomas [9], using a locking plate system $\left(\mathrm{TOM}^{\circledR}\right.$ plate) compared with a conventional crossed screw fixation. The Thomas technique was chosen on the one hand because the $\mathrm{TOM}^{\circledR}$ plate was especially designed for this lateral displacement osteotomy (Fig. 2b). In addition to this it represents an easily understandable technique with reliable results.

The hypothesis was that locking plate fixation provides more stability than srew fixation. 


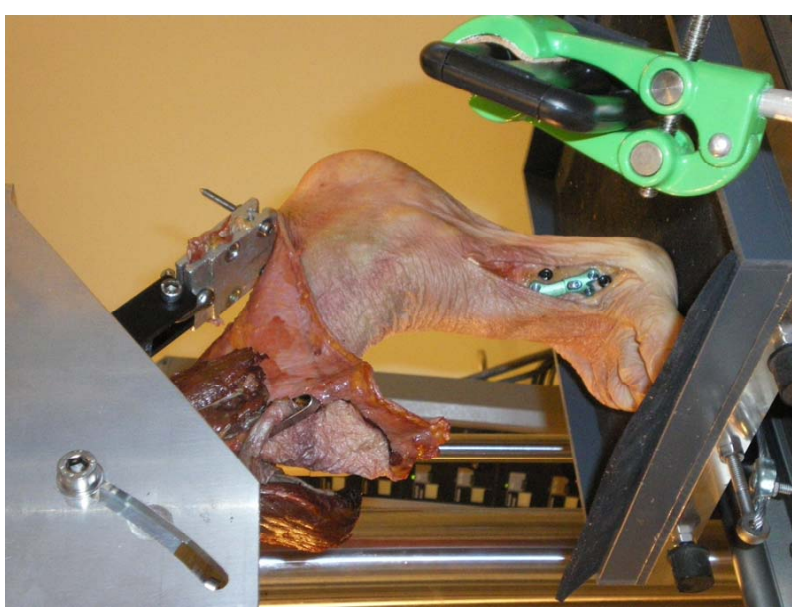

Fig. (1). Scheme of the experimental arrangement with specimen and Webcams. In the midst the specimen is seen clamped with the Achilles tendon to the MTS cylinder and touching the ground plate with the first metatarsophalangeal joint. The force of the MTS is applicated exactly over the forefoot to simulate the toe - off phase.

\section{MATERIALS AND METHODOLOGY}

\section{Test Model}

A biomechanical model was established in which, contrary to simplified representations using artificial bone or selected single bones of the forefoot, the complete anatomical human specimens were employed and a physiological stance phase with maximum load on the first metatarsal head was simulated. The design of the biomechanical model takes into consideration, that the maximum loading of the forefoot while walking occurs during the toe - off phase at the end of gait cycle. Fifty percent of the load under the forefoot in this phase of gait representing the second peak in the $\mathrm{M}$ - curve are distributed under the first MTP joint to the first metatarsal head, which thus corresponds to the maximum load on the osteosynthesis [10].

The relative movement of the first metatarsal osteotomy was measured after surgery and under loading as described below. Thus all two methods of fixation were loaded in the same way corresponding to a physiological load bearing of the first metatarsal of 50 percent with respect to the whole force applied to the forefoot at toe - off phase.

\section{Tested Specimens}

Seven anatomical human specimens were tested (average age 72, 1 male/ 6 female). The specimens were frozen after amputation, and thawed for 24 hours at room temperature before testing. All specimens were obtained from the American Institute of Biology (Science Care, 2020 West Melinda Lane). Ten specimens suffering from a hallux deformity with a metatarsus primus varus were avaiable. Due to the normal distribution of this deformity in the population predominated female specimens. Two were used for pre-testing and one was excluded from the evaluation for reasons explained below.

\section{Surgical Procedure}

Surgery was done according to the method described by Thomas [9] (Fig. 2a, b). Approximately $1.5 \mathrm{~cm}$ distal to the first tarsometatarsal joint line, a saw cut perpendicular to the axis of the first metatarsal was set completely through the bone and subsequently the distal fragment moved $2 \mathrm{~mm}$ in a lateral direction, so that this enabled a correction of the increased first intermetatarsal angle. The first tarsometarsal joint itself was left intact correcting only the varus deviation of the first metatarsal by lateral displacement. Due to the rectangular arrangement of screws when fixing the osteotomy with the locking plate the bone surface of the osteotomy was left intact. Moreover the bone structure was not compromised in respect to the oblique passage of the crossed cacellous screws.

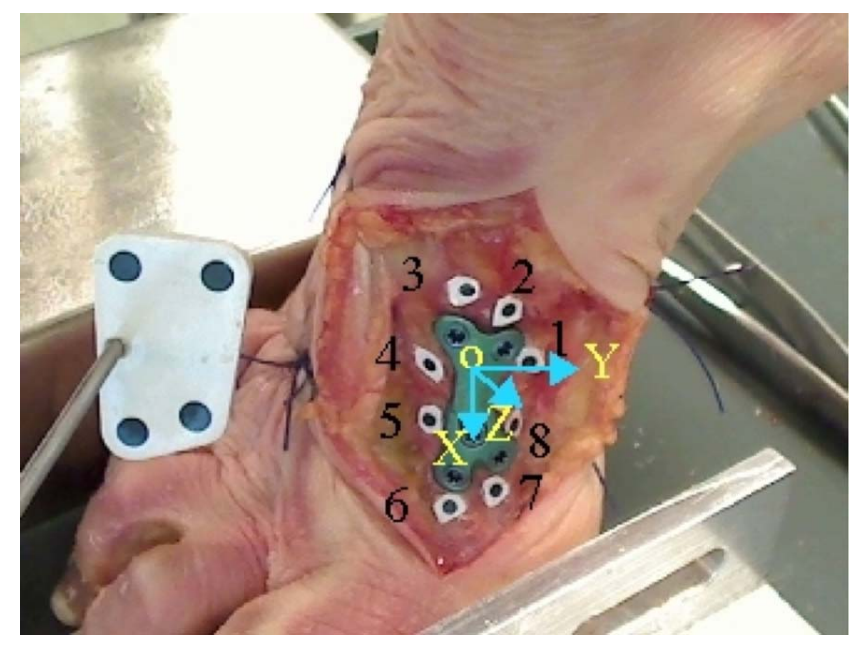

Fig. (2a). Arrangement of markers in the specimen and name of measurement axes. To record the relative movements on each side of the osteotomy four markers (diameter $2 \mathrm{~mm}$ ) were fixed, so that the change of the position of the proximal respective to the distal markers could be recorded alongside the $\mathrm{x}-\mathrm{y}-\mathrm{y}-$ and $\mathrm{z}-$ axis. Here, the $\mathrm{x}$ - axis runs from proximal to distal parallel to the first metatarsal, the $y$ - axis parallel to the osteotomy and perpendicular to the first metatarsal axis and the $\mathrm{z}$ - axis perpendicular to the plane definded by the markers $1-4$.

Based on the hypothesis that the locking plate fixation of the osteotomy is more stable than the crossed screws osteosynthesis and due to the results of pre-testing mentioned below, the angle-plate was installed first in order to prevent irreversible damage of the specimens and allow subsequent testing with the screw fixation. The locking screws were directed perpendicular to the first metatarsal bone whereas the crossed cancellous screws were placed in an oblique direction. Moreover the crossed cancellous srew fixation was done before unscrewing the plate. Thus it was guaranteed that the osteosynthesis stability was not compromised by using the same specimens for two different devices.

Each specimen was tested with a locking screw plate (PLATE) as well as a crossed cancellous srew fixation (CSO). Taking into consideration the results of the pre-tests where a load bearing of more than $200 \mathrm{~N}$ has caused breakage of the screw fixation with irreversible bone 


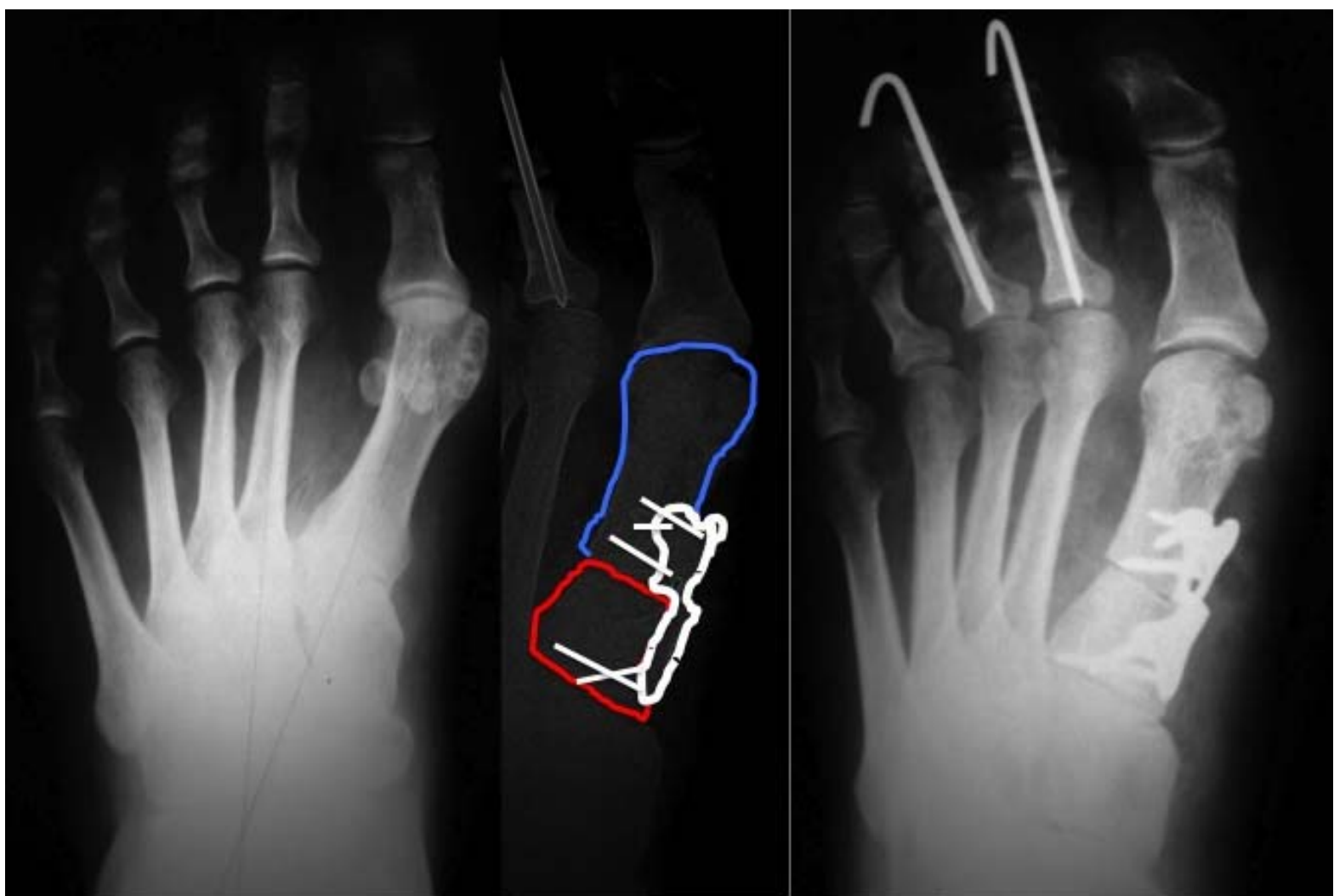

Fig. (2b). Preoperative X-ray, schematic drawing and postoperative X-ray of a proximal metatarsal lateral displacement osteotomy with a $\mathrm{TOM}^{\circledR}$ plate osteosynthesis.

destruction the plate fixation was done first. The cadaver specimens were amputated at the transition of lower to middle third of the tibia. The proximal tibial stump was fixed in a cylinder using cold-curing resin (Ureol FC 53, Vantico $\mathrm{GmbH}$, Wehr, Germany). The extrensic muscle tendons were fixed in this cylinder as well. Only the Achilles tendon was prepared and attached to a clamping device, which was firmly attached to the test-setup and allowed the application of the force in physiological vectorial direction (Fig. 1).

\section{Devices for Osteosynthesis}

As locking plate the TOM ${ }^{\circledR}$ plate device (Wright Medical Technique) was used. The device is designed such that a distal dynamic compression hole allows for the possibility of additional interfragmentary compression. To achieve this the plate is occupied by fixed angle screw fixation $(\varnothing 2.7 \mathrm{~mm})$ in the proximal osteotomy fragment, then a standard screw is applied in the DC - hole in the distal fragment first. This results in interfragmentary compression. Only then the distal osteotomy part is fixed to the plate with fixed angle screws. The plate is machined from a titanium alloy block. The rectangular step situated in the midth of the plate allows the lateral displacement of the distal fragment. Because of its design as a third tubular plate it is appropriate for medial first metatarsal application, so that a dorsal plate position with the risk of plantar gapping under load is avoided as well as the elaborate preparation necessary for a plantar plate system. As far as the authors know this plate is the only device enabling a combination of interfragmentary compression with locking screw fixation.
For screw fixation, standard $3.2 \mathrm{~mm}$ cancellous screws were used with a two third thread design. Care was taken that the standard screws were not crossing the screw holes of the $\mathrm{TOM}^{\circledR}$ plate what could lead to reduced stability of the osteosynthesis. That is why the standard screws were applied directed against each other and crossing the metatarsal osteotomy before the PLATE was taken off. Thus full bone contact was granted.

\section{Experimental Arrangement and Protocol}

\section{Arrangement}

After implantation of the device, the specimens were mounted in a Mini Bionics MTS testing machine. This was carried out in such a way that the first metatarsophalangeal joint was positioned exactly under the vectorial axis of the MTS cylinder based on a plate rectangular to the cylinder axis. The extrensic muscle tendons were fixed in the cylinder under mid - tension, the Achilles tendon was clamped on the MTS cylinder to simulate a physiological vectorial alignment, such that under loading of the forefoot a resisting tension on the tendons was created. The position of the tendon fixation could be changed so that during forefoot loading the plantar-flexion angle of the foot could be maintained under a predetermined angle depending on the individual shape of the specimens. The ankle positioning during testing was done according to its physiological position at terminal stance during gait. Thus the vectorial direction of the force exerted by the MTS was always situated exactly on the first metatarsophalangeal joint. 
The foot was mounted unconstrained with the exception of the holding force of the cylinder and of the Achilles tendon, and the ground-reaction and friction force on the forefoot. The axial load of the MTS cylinder could be increased with the ground reaction force under the first metatarsophalangeal joint corresponding to fifty percent of the load applied to the forefoot (Fig. 1). This arrangement allowed to simulate the toe - off phase of gait corresponding to a maximum of ground reaction force under the first MTP joint. An axial force of 20 Newton was first applied to the specimens so that ground contact of the clamped specimens to the base-plate occured. The axial force was subsequently continually increased, starting from the $20 \mathrm{~N}$ of force preload, to $50 \mathrm{~N}$, and then in further intervals of $50 \mathrm{~N}$ up to $200 \mathrm{~N}$. By using only submaximal axial forces, on the one hand the irreversible damage of the specimens should be avoided. In addition, the $200 \mathrm{~N}$ of applied load approximately corresponds to the amount of load the patients are allowed to apply postoperatively while only partial weight bearing of the foot is possible. At this time the osteosynthesis is most vulnerable as no bone healing has taken place. The model takes into consideration this critical phase where a stable osteosynthesis is essential for the success of the surgery.

\section{Protocol}

The relative movements of the osteotomy fragments to one another were measured using a steriometric optical measuring system which had been validated to have an accuracy of $\pm 0.1 \mathrm{~mm}$. Two webcams (Logitech ${ }^{\circledR}$, Webcam Pro 9000 , resolution $1600 \times 1200$ pixels) and a reference body (four black circles with a diameter of $5 \mathrm{~mm}$ on a white square) attached to a pin ( $27 \mathrm{~cm}$ long) between the cameras were installed on a tripod in such a way that the camera field of both cameras during the entire measuring process did not change and the position and the focus of the webcams remained fixed on the ROI, i.e. the osteotomy fragments (Fig. 2a). To record the relative movements on each side of the osteotomy four markers (diameter $2 \mathrm{~mm}$ ) were fixed, so that the change of the position of the proximal respective to the distal markers could be recorded alongside the $\mathrm{x}-, \mathrm{y}-$ and $\mathrm{z}$ - axis. Here, the $\mathrm{x}$ - axis runs from proximal to distal parallel to the first metatarsal, the $y$ - axis parallel to the osteotomy and perpendicular to the first metatarsal axis and the $\mathrm{z}$ - axis perpendicular to the plane defined by the markers 1-4 (Fig. 2a). Both translational and rotational relative movements were considered [11, 12]. The programs used for this purpose were written with MATLAB (The MathWorks, Inc., Nattick, USA).

The analysis of the relative movements of the osteotomy fragments was done by increasing the load imposed by the MTS cylinder according to the protocol described above. Then, the experimental setup was repeated after previously replacing the locking plate with crossed two thirds cancellous screws (ø $3.5 \mathrm{~mm}$ ).

The statistical analysis was performed by comparing both the translational and rotational relative movements of locking plate fixation on the one hand and crossed cancellous screw fixation on the other as an expression of the achieved stability of fixation between the two metatarsal osteotomy fragments. An unifactorial analysis of variance should be done as the parameter to be tested was defined as the influence of the ground reaction force applied to the specimens on the osteotomy. As the data did not fulfill the assumption of normality implicit in the analysis of variance it was conducted using a two-way analysis of variance by ranks as described by Friedman. The level of significance was $\mathrm{p}<0.05$.

All data were collected and analyzed prospectively.

\section{RESULTS}

We found larger stability about all three axes of movement analyzed for the PLATE than the CSO, i.e. the relative movements observed were lower along the $\mathrm{x}$ - axis distal to proximal, the y - axis parallel to the osteotomy and the $\mathrm{z}$ - axis perpendicular to the plane defined by the markers 1- 4 for PLATE than CSO. A striking observation hereby is the superiority of the locking plate especially with high plantar load measurements (Fig. 3). When observing translational relative movements of the osteotomy fragments at a load application of $200 \mathrm{~N}$, the maximum values for CSO were $2.71 \mathrm{~mm}, 3.9 \mathrm{~mm}$, and $1.71 \mathrm{~mm}$ and along the $\mathrm{x}, \mathrm{y}$, and $\mathrm{z}$ - axis respectively. In contrast, the PLATE shows maximum relative movements of only $0.66 \mathrm{~mm}, 0.9 \mathrm{~mm}$ and $0.35 \mathrm{~mm}$, again along the $\mathrm{x}, \mathrm{y}$ and $\mathrm{z}-$ axis respectively. Correspondingly, the PLATE shows maximal relative rotational movement at $200 \mathrm{~N}$ load of $0.9^{\circ}, 2.36^{\circ}$, and 2.28 $\circ$, and a $7.1^{\circ}, 9.1^{\circ}$, and $2.71^{\circ}$ for the CSO about the three axes respectively.

The two - way analysis of variance by ranks done with the results of the $\mathrm{x}, \mathrm{y}$ and $\mathrm{z}$ groups for PLATE and CSO showed statistical significance at a level of $\mathrm{a}=0.05$ for all groups and both translational and rotational movements for a load from $20 \mathrm{~N}$ to $200 \mathrm{~N}$ for all three axes (Fig. 3).

The osteosythesis with crossed cancellous screws failed macroscopically in one case when the specimen was loaded with more than $200 \mathrm{~N}$. Therefore this specimen was not included in the statistical analysis. In all other cases (seven specimens) an osteosynthesis failure at the plate-screw interface, as a failure of the fixed threaded screw head or as a patent screw or plate fracture was not observed.

\section{DISCUSSION}

This study demostrates the superior stability of the locking plate fixation compared to the crossed cancellous screw osteosynthesis.

Both in terms of translational and rotational relative movement of the osteotomy fragments, the locking plate was observed to be more stable under the application of a ground reaction force under the first metatarsal head of $200 \mathrm{~N}$. The locking plate resulted in a significantly less relative movement of the osteotomy fragments with respect to each other.

Using proximal first metatarsal osteotomies, a very high potential of correction can be realized so that severe hallux valgus deformities can also be corrected and sufficiently stabilized. Essentially four different methods are mentioned in literature: closing - wedge osteotomy, opening - wedge osteotomy, crescentic osteotomy with curved cutting incision and proximal Chevron - osteotomy [13, 14]. For all of these methods, it could be shown that conventional methods of 
Translation, $\mathrm{x}$-axis, $\mathbf{m m}$

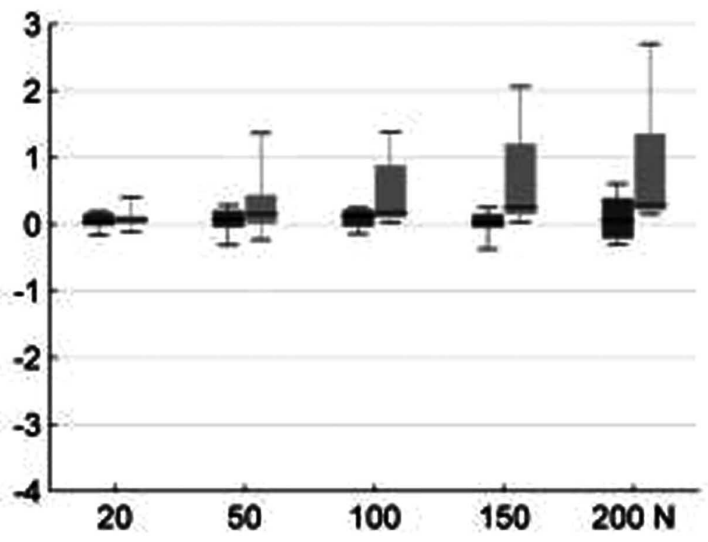

Translation, $\mathbf{y}$-axis, $\mathbf{m m}$

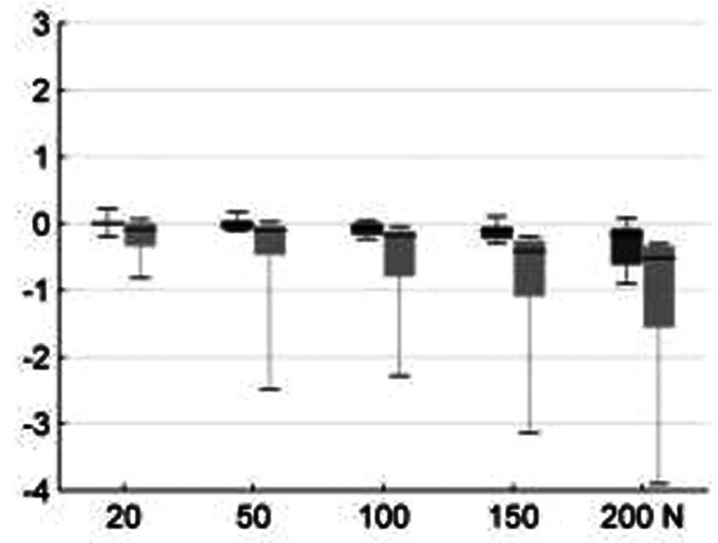

Translation, $\mathrm{z}$-axis, $\mathbf{m m}$

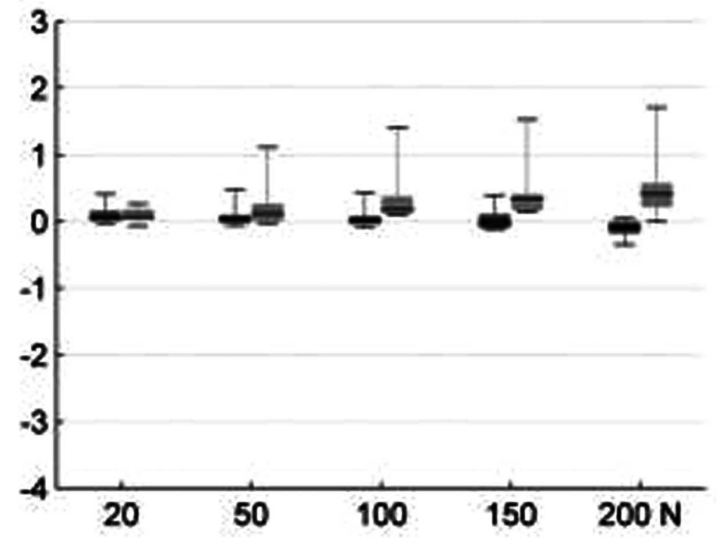

Rotation, $\mathrm{x}$-axis, degree

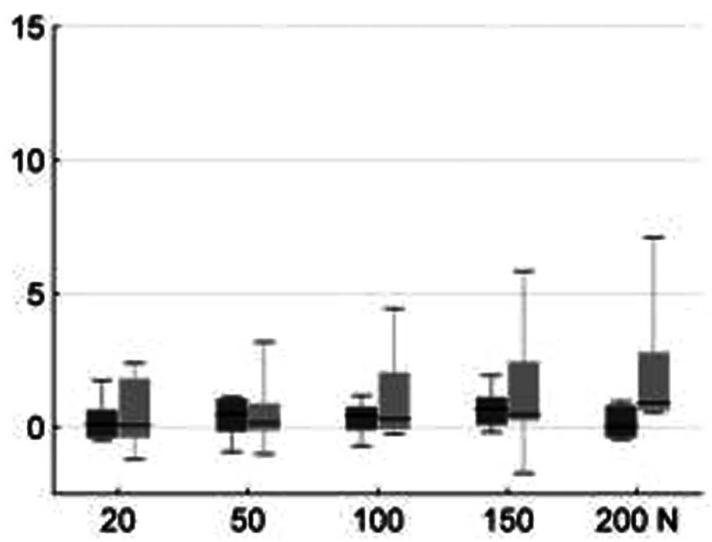

Rotation, y -axis, degree

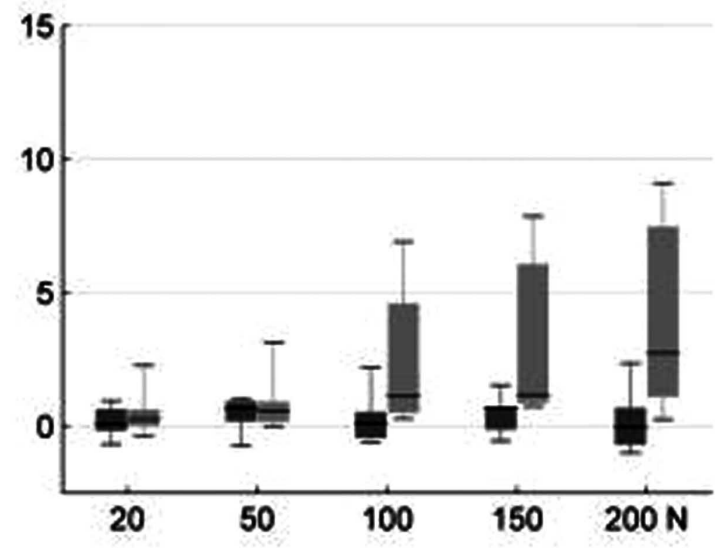

Rotation, $\mathrm{z}$-axis, degree

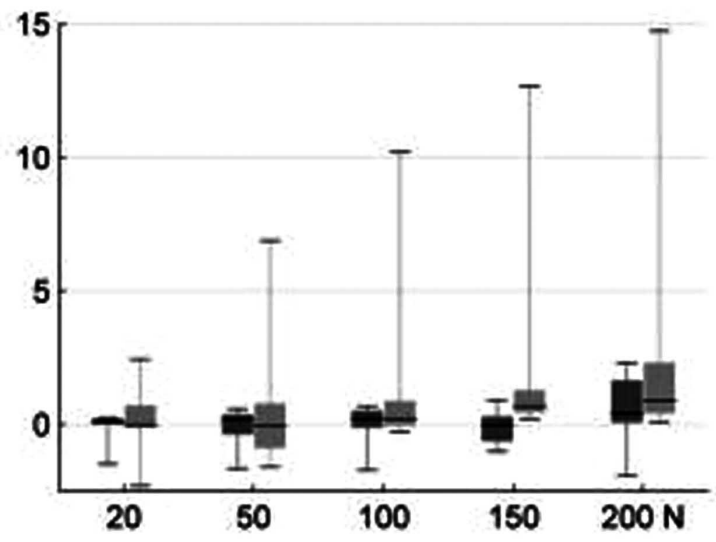

Fig. (3). Relative fracture fragment motion measured for the PLATE and CSO. Shown is a boxplot diagram for relative movements of the osteotomy fragments in millimeter rsp. degree (mean indicated by small black line). 
osteosynthesis like screw, plate or wire fixation are much less stable than for distal correction procedures [15]. A stable internal fixation however is essential to prevent a pseudarthrosis or secondary loss of correction.

Campbell et al., as well as Rosenberg et al., demostrated that a conventional plate osteosynthesis is superior on stability than screw fixation $[16,17]$.

Some of these procedures show well known disadvantages: shortening of the first ray with the risk of developing a transfer metatarsalgia (closing wedge osteotomy) or an increase in the already pathologically enlarged DMMA angle (opening wedge osteotomy) what requires an additional distal metatarsal osteotomy. Crescentic osteotomies last but not least are technically demanding and require high surgical skill [5]. All this is avoided by a lateral displacement osteotomy in the Thomas technique, especially since a wide variability of possible saw cuts is tolerated by the procedure, both perpendicular to the shaft as well as descending or ascending [9]. A stable internal fixation, however, is mandatory as well.

The failure of internal fixation may be realized either in a single event with maximum load application, e.g. in a fall, or in terms of a gradual failure of load application in the sense of fatigue. The latter can either lead to a pseudarthrosis or displacement of the distal fragment, especially in dorsiflexion $[18,19]$. A displacement rate as high as $28 \%$ of the patients with a first ray dorsiflexion after a crescentic osteotomy is reported in literature $[19,20]$.

For internal fixation most often crossed screws, plate osteosynthesis or additional Kirschner wires are recommended [17, 21-23]. Compared to these techniques, a locking plate system has several advantages. It protects the blood supply of the bone and offers a much higher stability of the fixation of the osteotomy fragments one to each other [24]. So it was only obvious that the principle of locking plate fixation was applied on first metatarsal osteotomies as well. Nevertheless Cohen found no benefits of the locking plate system compared to crossed screw fixation in the first tarsometatarsal joint arthrodesis [25]. These findings however may be due to the specific design of the locking plate consisting of a relatively soft titanium alloy without possibility of interfragmentary compression and obligatory fixation at the dorsal side of the first metatarsal with the risk of plantar osteotomy gapping. The $\mathrm{TOM}^{\circledR}$ plate used in our study is made of a more solid titanium alloy, facilitates interfragmentary compression and is placed at the medial side of the MT 1 . The benefits of interfragmentary compression with increased bone contact and maximum friction between the bone surfaces can thus be combined with the increased rigidity and stability of the locking plate system.

In this investigation, the stability of the $\mathrm{TOM}^{\circledR}$ plate was compared to CSO in a complete human cadaver-foot model, which does not rely on isolated bone specimens or artificial bone substitute materials. A complex plantar dissection necessary to improve the stability by a plantar plate placement [26] was not necessary. Test models described in literature often use feet disarticulated in the ankle, arranged in an unphysiological position, measuring only translational forces or rely on synthetic bone [25, 27-29]. In some studies the locking plate lacked the possibility of interfragmentary compression and three - dimensional aspects were not taken into consideration in contrast to our study [30,31].

To our knowledge, our study is the only one in which a biomechanical model was established which does not rely on simplified representations and where the complete anatomical human specimen was employed and a physiological position and loading were applied simulating the in vivo loading situation. The maximum loading condition of the first metatarsophalangeal joint applied in this study has for instance been documented by means of dynamic pedobarography, where this phase of the stancephase of gait is correlated to peak pressures and loading [10, 32-35]. By using the same specimens to test both osteosynthesis techniqes the bias resulting from specimen variability could be reduced and the stability of the implants compared in a paired experimental design. As mentioned above, care was taken to avoid that the standard screws were crossing the screw holes of the $\mathrm{TOM}^{\circledR}$ plate leading to reduced stability of the osteosynthesis. To grant full bone contact the standard screws were applied directed against each other and crossing the metatarsal osteotomy before the plate fixed with locking screws was taken off. The locking screws fixed to the plate were just staying in the first metatarsal when the standard screws were appplied. Therefore the standard screws were placed in independent holes.

The authors' decision to compare the stiffness of locking plate fixation with that of standard screw fixation was done because they wanted to refer to a basic and well established method of osteosynthesis for metatarsal osteotomies $[13,16$, $23,25]$.

The measuring equipment and methods had been validated before the actual tests in a pre-test setting. In contrast to the studies mentioned above a three - dimensional testing model was used. Thus the focus of measurement was not fixed to a single plane whereas a possible failure of the fixation in one of the other planes occurred. Rather the three - dimensional orientation of the ostoetomy and the osteosynthesis was analysed with our testing model taking into consideration both rotational and translational forces.

The situation of measurement corresponds to the commonplace situation before surgery is done. Normally a bone mineral density of the foot is not available for the patient. Therefore the possible results cannot be taken into consideration resulting in the requirement of the most stable osteotomy fixation in respect of the assumption of poor bone quality.

\section{CONCLUSION}

The results of our study confirmed that the lateral proximal first metatarsal displacement osteotomy in combination with a locking plate fixation delivers several advantages at once. On the one hand, it avoids the drawbacks of other first metatarsal osteotomies, such as shortening of the first ray or a technically difficult exposure of the ROI with a high skilling factor and corresponding risks. On the other hand, the TOM ${ }^{\circledR}$ locking plate, which also allows internal fragment compression, is a possibility of a technically simple internal fixation of superior stability compared to other osteosynthesis devices. 
The results of this study suggest that a permanent deformity correction without postoperative relative movements can be realized, which is prerequisite for reliable bone healing. In our view, the locking plate device investigated thus provides due to its design with the combination of interfragmentary and locking screw fixation the high internal stability necessary for an early functional treatment after hallux valgus correction, while avoiding the otherwise necessary period of prolonged immobilization.

\section{ACKNOWLEDGEMENT}

Declared none.

\section{CONFLICT OF INTEREST}

Declared none.

\section{REFERENCES}

[1] Craigmile DA. Incidence, origin and prevention of certain foot defects. Br Med J 1953; 2: 749-52.

[2] Gottschalk FAB, Sallis JG, Solomon L, Beighton PH. A comparison of the prevalence of hallux valgus in three African populations. J Bone Joint Surg 1979; 61 - B: 245-55.

[3] Kilmartin TE, Barring RL, Wallace WA. Metatarsus primus varus, a statistical study. J Bone Joint Surg 1991; 73-B: 937-40.

[4] Kelikian H. Hallux valgus, allied deformities of the forefoot and metatarsalgia. Philadelphia: WB Saunders 1965; pp. 1-5.

[5] Mann RA, Coughlin MF. Adult hallux valgus. In: Coughlin MJ, Mann RA Eds. Surgery of the foot and ankle. St. Louis: Mosby 1999; pp. 150-269.

[6] Trnka HJ, Muhlbauer M, Zembsch A. Basal closing wedge osteotomy for correction of hallux valgus and metatarsus primus varus: 10 to 22 years of follow-up. Foot Ankle Int 1999; 20: 171-7.

[7] Hallux valgus AWMF Leitlinien. 2009; Available from: http://leitlinien.net

[8] Wülker N. Hallux valgus - Hallux rigidus. Stuttgart: Enke Verlag 1997; p. 95.

[9] Thomas M. Die proximale Verschiebeosteotomie mit winkelstabiler Plattenosteosynthese zur Korrektur der fortgeschrittenen Hallux valgus Deformität. Fuß Sprunggel 2009; 1: 14-21.

[10] Hansen STJ. Functional reconstruction of the foot and ankle. Philadelphia, Baltimore, New York: Lippincott Williams and Wilkins 2000.

[11] Bobrowitsch E, Imhauser C, Graichen H, Dürselen L. Evaluation of a 3D object registration method for analysis of humeral kinematics. J Biomech 2007; 40: 511-8.

[12] Bobrowitsch E, Hurschler C, Olender G, et al. Digital stereophotogrammetry based on circular markers and zooming cameras: evaluation of a method for 3D analysis of small motions in orthopaedic research. Biomed Eng Online 2011; 10-12. Available from: http://www.biomedical-engineering-online.com

[13] Acevedo JI. Fixation of metatarsal osteotomies in the treatment of hallux valgus. Foot Ankle Clin 2000; 5: 451-68.

[14] Trnka HJ, Parks BG, Ivanic G. Six first metatarsal shaft osteotomies: mechanical and immobilization comparisons. Clin Orthop 2000; 381: 256-65.

[15] Shereff MJ, Sobel MA, Kummer FJ. The stability of fixation of first metatarsal osteotomies. Foot Ankle Int 1991; 11: 208-11.

[16] Campbell JT, Schon LC, Parks BG, Wand Y, Berger BI. Mechanical comparison of biplanar proximal closing wedge osteotomy with plantar plate fixation versus crescentic osteotomy with screw fixation for the correction of metatarsus primus varus. Foot Ankle Int 1998; 19: 293-9.

[17] Rosenberg GA, Donley BG. Plate augmentation of screw fixation of proximal crescentic osteotomy of the first metatarsal. Foot Ankle Int 1998; 19: 430-7.

[18] Acevedo JI, Sammarco VJ, Boucher HR. Mechanical comparison of cyclic loading in five different first metatarsal shaft osteotomies. Foot Ankle Int 2002; 23: 711-6.

[19] Mann RA, Rudicel S, Graves SC. Repair of hallux valgus with a distal soft - tissue procedure and proximal metatarsal osteotomy. A long-term follw-up. J Bone Joint Surg 1992; 74 - A: 124-9.

[20] Thordason DB, Leventen EO. Hallux valgus correction with proximal metatarsal osteotomy: two - year follow -up. Foot Ankle Int 1992; 13: 321-6.

[21] Amis JA, Porter DA. Correction augmentation and provisional fixation in proximal metatarsal osteotomies using Kirschner wires. Foot Ankle Int 1999; 20: 752-3.

[22] Anderson RB, Davis WH. Internal fixation of the proximal chevron osteotomy. Foot Ankle Int 1997; 18: 371-2.

[23] Toomey EP, Mc Garvey SR. Fixation of the proximal first metatarsal crescentic osteotomy with the small cannulated screw system. Foot Ankle Int 1991; 11: 397-9.

[24] Schütz M, Südkamp NP. Revolution in plate osteosynthesis: new internal fixation system. J Orthop Sci 2003; 8: 252-8.

[25] Cohen DA, Parks BG, Schon LC. Screw fixation compared to H Locking plate fixation for first metatarsocuneiform arthrodesis: a biomachanical study. Foot Ankle Int 2005; 26: 984-9.

[26] Marks RM, Parks BG, Schon LC. Roger A. Mann Award. Midfoot fusion technique for neuropathic feet: biomechanical analysis and rationale. Foot Ankle Int 1998; 19: 507-10.

[27] Kimball HL, Aronow MS, Sullivan RJ, Tarinelli DJ, Nowak MD. Biomechanical evaluation of Calcaneocuboid distraction arthrodesis:a cadaver study of two different fixation methods. Foot Ankle Int 2000; $21: 845-8$.

[28] Politi J, Haye J, Njus G, Bennett GL, Kay DB. First metarsal phalangeal joint arthrodesis: a biomechanical assessment of stability. Foot Ankle Int 2003; 24: 332-7.

[29] Neufeld SK, Parks BG, Naseef GS, Melamed EA, Schon LC. Arthrodesis of the first metarsophalangeal joint: a biomechanical study comparing memory compression staples, cannulated screws, and a dorsal plate. Foot Ankle Int 2002; 23: 97-101.

[30] Hofstaetter SG, Glisson RR, Alitz CJ, Trnka HJ, Easley ME. Biomechanical comparison of screws and plates for hallux valgus opening - wedge and Ludloff osteotomies. Clin Biomech 2008; 23(1): 101-8.

[31] Scott AT, DeOrio JK, Montijo HE, Glisson RR. Biomechanical comparison of hallux valgus correction using the proximal chevron osteotomy fixed with a medial locking plate and the Ludloff osteotomy fixed with two screws. Clin Biomech 2010; 25(3): 2716.

[32] Betts RP, Frank CI, Duckworth T. Foot pressure studies: normal and pathological gait analysis. In: Jahss $\mathrm{MH}$, Ed. Disorder of the foot and ankle: medical and surgical management. $2^{\text {nd }}$ ed. Philadelphia: WB Saunders 1991; pp. 484-579.

[33] Harrison AJ, Folland JP. Investigation of gait protocols for plantar pressure measurement of non - pathological subjects using a dynamic pedobarograph. Gait Posture 1997; 6: 50-7.

[34] Henning EM, Milani TL. Die Dreipunktunterstützung des Fußes: eine Druckverteilungsanalyse bei statischer und dynamischer Belastung. Z Orthop Grenzgeb 1993; 131: 279-84.

[35] Henning EM, Staats A, Rosenbaum D. Plantar pressure distribution patterns of young school children in copmarison to adults. Foot Ankle Int 1994; 15: 35-40. 